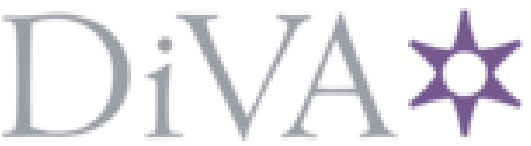

http://www.diva-portal.org

Postprint

This is the accepted version of a paper presented at 2018 IEEE OES Autonomous Underwater Vehicle Symposium.

Citation for the original published paper:

Bore, N., Torroba, I., Folkesson, J. (2018)

Sparse Gaussian Process SLAM, Storage and Filtering for AUV Multibeam Bathymetry In: 2018 IEEE OES Autonomous Underwater Vehicle Symposium

N.B. When citing this work, cite the original published paper.

Permanent link to this version:

http://urn.kb.se/resolve?urn=urn:nbn:se:kth:diva-250895 


\title{
Sparse Gaussian Process SLAM, Storage and Filtering for AUV Multibeam Bathymetry
}

\author{
Nils Bore, Ignacio Torroba \& John Folkesson \\ Robotics, Perception and Learning Lab, KTH, Stockholm \\ \{nbore, torroba, johnf\}@kth.se
}

\begin{abstract}
With dead-reckoning from velocity sensors, AUVs may construct short-term, local bathymetry maps of the sea floor using multibeam sensors. However, the position estimate from dead-reckoning will include some drift that grows with time. In this work, we focus on longterm onboard storage of these local bathymetry maps, and the alignment of maps with respect to each other. We propose using Sparse Gaussian Processes for this purpose, and show that the representation has several advantages, including an intuitive alignment optimization, data compression, and sensor noise filtering. We demonstrate these three key capabilities on two real-world datasets.
\end{abstract}

\section{INTRODUCTION}

As Autonomous Underwater Vehicles (AUVs) become more capable, they carry out longer missions, and with new sensors, they can survey the sea floor with increasing precision. Both factors contribute to larger amounts of data being produced in a single AUV deployment. For intelligent AUV localization and path planning, it is critical to maintain and utilize as much information as possible from the collected data. As an example, precise Terrain-Aided SLAM [1] uses the full geometry of the observed sea floor for localization, requiring the storage of fused bathymetry data from a potentially long deployment. Likewise, optimal 3D path planning requires the full geometry of the environment to be known. With the limited processing capability onboard most AUVs, these applications require summarization and compression of bathymetry data.

In this work, we propose a single, cohesive representation, which achieves the following goals:

- $\quad$ to summarize the data by aligning local maps into a larger global map,

- to compress the bathymetry data by fitting several 2D surfaces,

- $\quad$ and to remove unnecessary data points by filtering sensor noise.

The fulfillment of these goals could extend the deployment time of an AUV and its operation robustness. By aligning local multibeam maps produced by deadreckoning (DR), the AUV may limit the unbounded growth of DR positioning. The compression of the bathymetry data allows us to maintain high-precision maps of the covered sea floor for extended periods. Finally, filtering increases the precision of the maps and improves robustness of algorithms operating on the data.

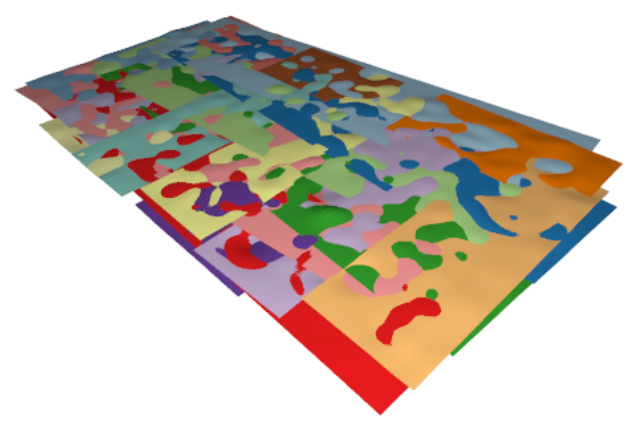

Fig. 1: We represent the sea floor bathymetry using continuous Gaussian process submaps, which can also be jointly optimized to produce a SLAM solution. Here, each colored area corresponds to one GP submap.

The representation proposed in this work is Sparse Gaussian Processes [2], see Figure 1. Gaussian processes (GPs) have been successfully used as a representation for bathymetry data, most notably in [3], where GPs are used to train the measurement model of a particle filter SLAM framework. In our work, we employ GPs as the sole map representation. By using the sparse online GP method of [2], we do not need to retrain the representation as new multibeam measurements are coming in, as in [3]. In addition, the method sparsifies the GP bathymetry within an allowed error margin, enabling compression of the data. By employing a sparse factor graph framework similar to [1], we also ensure that our alignment optimization stays feasible as we collect more data. Our novel registration method is defined in a probabilistic manner and performed online during factor graph optimization. In experiments, we quantify the advantage of our compression and filtering, in addition to alignment results.

We present the following contributions:

- A novel, compact bathymetry representation that allows large-scale bathymetric SLAM

- A factor-graph SLAM formulation that registers 
bathymetry submaps online as part of the global map optimization

\section{RELATED WORK}

Bathymetric maps have long been used for ships to safely navigate in shallow waters. In more recent autonomous sea vessels, Terrain-Aided Navigation (TAN) [4] represents an early example of their use for vehicle navigation. The idea of TAN is to localize the vehicle with respect to a pre-defined sea floor elevation map. By comparing the current altitude measurements to the map, a TAN system can draw conclusions about the vehicle location. The methodology has been employed in numerous applications, for example using single-beam altimeters in gliders [5]. Most modern TAN systems implement a probabilistic model as suggested by Nygren and Jansson [4]. To provide a realistic measurement model even when the prior bathymetric map is coarse, Zhou et al. [6] proposed modeling the underlying sea floor surface as a Gaussian Process. With additional priors on the sea floor shape, they demonstrate that TAN can function even in this more challenging scenario.

If a vehicle is to explore new areas, it can not rely on prior maps. Instead, it needs to perform simultaneous localization and mapping (SLAM). Several works in the last decade have focused on featureless methods for bathymetric SLAM. Roman and Singh [7] were among the first to propose the construction of bathymetry submaps. Each submap comprise some sequence of subsequent multibeam measurements transformed into a common reference frame using the dead-reckoning estimate. They identified a key trade-off when determining the size of the submaps; they must be large enough to align to one another, and small enough as not to include significant DR drift within the submaps. Similar to many later works, they used the iterative-closest point (ICP) algorithm to align submaps, and then included the computed transformation in a factor-graph optimization. More recently, Bichucher et al. [1] improved upon this framework by incorporating a more powerful Generalized ICP algorithm, which also provides more realistic transformation covariances which are used in the factorgraph optimization. There has also been work to use similar methods for inspection of complex structures [8]. In that scenario, the authors take care to limit the along-track beam spread of the scanning sonar. By filtering the walter-column image they produce more reliable range detections. The submaps are then formed by fusing individual detections in a volumetric voxel grid, from which points can be extracted by a voxel confidence threshold. Again, the filtered submaps are aligned similar to [7].

While the algorithms previously discussed may be characterized as smoothing methods, another class of algorithms for bathymetric SLAM is based on probabilistic filtering. Barkby et al. [9] introduced the $B P$ Slam algorithm, which is a Rao-Blackwellized particle filter, wherein the map is sampled and the pose of the vehicle is tracked analytically for each particle using an EKF filter. In subsequent papers [10] [3], the authors extended the method with a probabilistic measurement model based on Gaussian processes. The filter keeps a record of all measurements, and constructs a GP for each particle that can then be used for regression and comparison with new measurements. Similar to Zhou [6], the authors argue that such a model may be used to compare submaps with nearby, but unobserved areas. Another interesting extension of BPSlam to handle mapping of drifting ice bergs was recently proposed in [11].

Historically, elevation grids [9] and point clouds [7][1] have been the main map representation in bathymetric SLAM. As we have seen, more recently [6] and to some extent [10][3] have instead proposed use Gaussian processes to represent the sea floor elevation. However, there are also other, more exotic representations such as the signed distance function [12]. While well known in the robotic and computer vision communities, it has thus far seen little use in subsea mapping. Similar to GPs, it can be seen as an implicit surface representation, and the authors argue that it is well suited to filter noise in complex geometries such as in the inland mine scenario discussed in the paper [12].

Unlike previous works on submap SLAM with factor-graph optimization [7][1][8], we propose performing registration of the submaps online, at the same time as the optimization. This may prevent the registration of individual pairs from getting stuck in local minima, making the overall algorithm more robust. In particular, this may be a problem in scenarios with significant dead-reckoning drift, giving poor initial transform guesses for registration. In addition, we argue that our sparse GP representation is more compact than other bathymetric maps such as point clouds or voxel grids, while allowing for a high level of detail. This claim is verified by our results, and the resulting system should allow online mapping of large areas of sea floor.

\section{METHOD}

In our scenario, an AUV is surveying an area and collecting multibeam measurements of the sea floor. Using dead-reckoning, it estimates its position and constructs local multibeam maps of smaller areas along its way. We will refer to such maps as submaps. Each submap $i$ comprises concatenated multibeam 3D point measurements $\mathcal{P}_{i}=\left\{\mathbf{p}_{1}, \ldots, \mathbf{p}_{N_{i}}\right\}$, expressed in coordinates defined by a submap transform $\mathbf{T}_{i}$. Our method aims to compress these submaps in a principled manner, and to align them in a global coordinate system. In this setting, alignment amounts to adjusting the initial DR transforms $\mathbf{T}_{i}$ to ensure geometric consistency between the overlapping submaps. As noted in previous work [7], we need to choose the size of the submaps in such a way that they contain enough information to be aligned, 
at the same time as not including too much drift from DR. In this work, we will assume that there is already a scheme for dividing measurements into submaps. One may use methods proposed in previous work such as [7][1]. In addition, the maps may contain noise such as false returns e.g. from multi-path scattering or crosstalk. Our method also aims to remove such noise from the individual submaps by enforcing a local geometric consistency model.

\section{A. Sparse Gaussian Processes}

Gaussian processes (GPs) are a family of continuous stochastic processes. In our case, we use them to model the elevation at each point in our 2-dimensional $x y$ submap space. The prediction of the GPs will be the submap elevation $z$ at the point. General GPs are defined by their training points, sometimes called basis vectors. In our case, the training points are the $3 \mathrm{D}$ submap points $\mathcal{P}_{i}$. By defining a kernel function $k$ (sometimes called a covariance function), we can use the basis vectors to predict $z$ at arbitrary $x y$ points, together with the estimate uncertainty. We will take $\theta_{i}$ to mean all parameters of submap $i$, including the kernel and basis vectors. The kernel function between two points $k\left(\mathbf{x}, \mathbf{x}^{\prime}\right)$ explicitly defines the respective covariance matrix entries of the two points. In particular, GP regression requires the evaluation of $k$ at the input point $\mathbf{x}^{\prime}$ together with the basis vectors that span the GP. With many added basis vectors, regression might therefore be overly costly.

Naively, it should be apparent that at some point the estimates will not be affected by adding more basis vectors to a part of the input space that is already well covered. The idea behind most sparse GP methods is therefore to discard training points if they do not contribute significant new information to the GP model. In our work, we use the sparse GP method proposed in [2], since it allows online training of the process as new data points are coming in from the sensor. However, most other methods for sparsifying GPs would likely also be compatible with our framework.

\section{B. Gaussian Process Likelihood \& Filtering}

Sparse GPs [2] enable us to compress the submap data, and provide mean and uncertainty estimates of sea floor height at each $x y$ coordinate. Our GPs include a model of the sensor noise, as well as a prior on the sea floor smoothness as specified by a squared exponential kernel. The model allows the formulation of the marginal likelihood $L(\mathbf{p} \mid \theta)$ of a point to originate from a GP with parameters $\theta$. The mean negative $\log$ likelihood of a set of points from an overlapping submap $\mathcal{P}_{2}$ w.r.t. the process of submap 1 is then given by

$$
N L L_{1}\left(\mathcal{P}_{2}, \mathbf{T}\right)=-\frac{1}{\left|\mathcal{P}_{2}\right|} \sum_{\mathbf{p} \in \mathcal{P}_{2}} \log L\left(\mathbf{p}(\mathbf{T}) \mid \theta_{1}\right)
$$

Minimization of $N L L_{1}\left(\mathcal{P}_{2}, \mathbf{T}\right)$ with respect to the transform $\mathbf{T}$ with a standard optimization technique such as gradient descent defines a fully workable registration method. In addition, we may use the likelihood for filtering noisy (i.e. highly non-smooth) multibeam measurements. Filtering is achieved by rejecting point measurements $\mathbf{p} \in \mathcal{P}_{i}$ with likelihood smaller than a threshold $\tau$ with respect to $i$ 's process parameters $\theta_{i}$.

\section{Motion Prior}

To take advantage of the precision of the AUV navigation sensors, our SLAM algorithm also incorporates a prior on the uncertainty of dead reckoning. We assume the position uncertainty after $1 \mathrm{~m}$ can be characterized by a Gaussian with covariance $Q_{1}$. We form the cumulative error within one submap length, that is, between the first vehicle position $\mathbf{f}_{i}$ of submap $i$ and the last position $\mathbf{l}_{i}$. If we assume that the AUV travels on a straight line within the submap, it is given by

$$
\mathbf{Q}\left(\mathbf{f}_{i}, \mathbf{l}_{i}\right)=\left\|\mathbf{f}_{i}-\mathbf{l}_{i}\right\|_{2} \mathbf{Q}_{1} .
$$

Note that the accumulated error $\mathbf{Q}\left(\mathbf{f}_{i}, \mathbf{l}_{i}\right)$ may also be estimated for example by an EKF filter, allowing for more complex tracks.

Within our optimization system we add a constraint between consecutive submaps, as given by the set $\mathcal{C}$ of consecutive submap indices $i, j$. Each prior constraint takes the form of a normal distribution between the last track point $\mathbf{l}_{i}$ and the first of the next submap $\mathbf{f}_{j}$. In the optimization, we minimize the negative likelihood of the Gaussian density, without the constant denominator, giving the negative log prior loss

$$
N L Q_{i}\left(\mathbf{f}_{j}, \mathbf{T}\right)=\frac{1}{2}\left(\mathbf{f}_{j}(\mathbf{T})-\mathbf{l}_{i}\right)^{T} \mathbf{Q}\left(\mathbf{f}_{i}, \mathbf{l}_{i}\right)^{-1}\left(\mathbf{f}_{j}(\mathbf{T})-\mathbf{l}_{i}\right)
$$

\section{Submap Alignment}

Our Gaussian Process Graph SLAM (GPGS) method combines constraints from overlapping maps as well as consecutive maps into a graph optimization. To this end, we form a global cost function $c$ that includes consecutive constraints as given by $\mathcal{C}$ and $N L Q_{i}$. Similarly, we assume that there is some method that returns the set of overlapping submap indices $\mathcal{M}=\left\{i, j ; \mathcal{P}_{i}, \mathcal{P}_{j}\right.$ overlap $\}$. The total contribution of all consecutive and overlapping submaps is given by

$$
\begin{aligned}
c\left(\mathbf{T}_{1}, \ldots, \mathbf{T}_{N}\right) & =\sum_{(i, j) \in \mathcal{M}} N L L_{i}\left(\mathcal{P}_{j}, \mathbf{T}_{i}^{-1} \mathbf{T}_{j}\right) \\
& +\sum_{(i, j) \in \mathcal{C}} N L Q_{i}\left(\mathbf{f}_{j}, \mathbf{T}_{i}^{-1} \mathbf{T}_{j}\right) .
\end{aligned}
$$

The global cost function $c$ may be thought of as a posterior approximation, including likelihood terms $N L L_{i}$ and priors $N L Q_{i}$.

An approximate solution to the minimization of $c$ is found using the least squares minimization tool Ceres [14]. As in [1], we constrain the optimization to the $x y$-position, leaving us with translations $\mathbf{T}_{i} \in \mathbb{R}^{2}$. The 


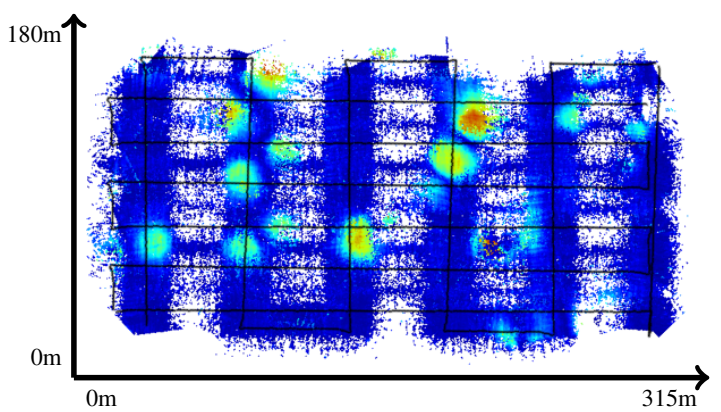

(a) Dead reckoning, misaligned pockmarks visible in red.

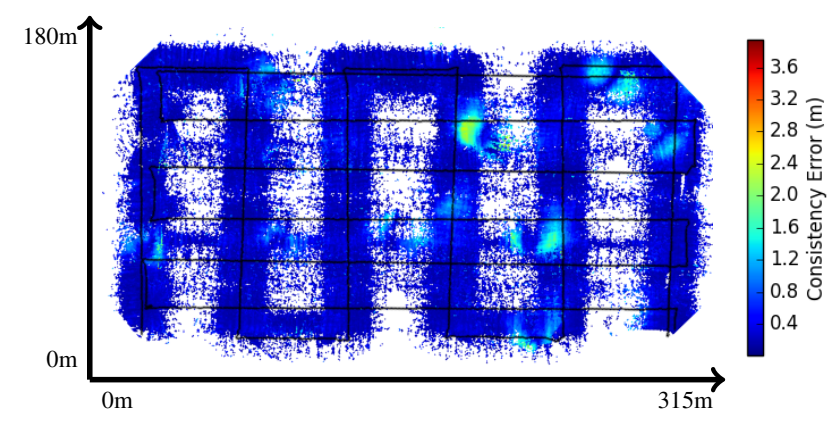

(b) GPGS slam result. Most of the error is removed.

Fig. 2: Consistency maps from 17 submaps in the pockmarks data set, with the error from [13]. Estimated tracks shown in black.

problem $\min _{\mathbf{T}_{1}, \ldots, \mathbf{T}_{N}} c\left(\mathbf{T}_{1}, \ldots, \mathbf{T}_{N}\right)$, with constraints on the transformations $\mathbf{T}_{i}$, is not a least squares optimization problem as presented above. However, with some minor tweaks it can be made into one. The prior terms $N L Q_{i}$ already take on a quadratic form, see Section III-C. But the likelihoods $N L L_{i}$ need to be altered slightly to fit into a least squares framework. In our case, we approximate the $L_{1}$ norm of the nonnegative scalar $N L L_{i}$ with the soft $L_{1}$ loss.

\section{E. Likelihood gradient}

Minimizing $c\left(\mathbf{T}_{1}, \ldots, \mathbf{T}_{N}\right)$ using a solver typically requires calculation of the gradients of $N L L_{i}$ and $N L Q_{i}$ with respect to the transform parameters $\mathbf{T}_{i}$. While differentiation of $N L Q_{i}$ is trivial and can often be done automatically by the solver, the likelihood gradient $\frac{\partial}{\partial \mathbf{T}_{i}} N L L_{i}$ warrants some discussion. Applying the gradient to the likelihood of Equation 1 gives us

$$
\frac{\partial}{\partial \mathbf{T}_{i}} N L L_{i}=-\frac{1}{\left|\mathcal{P}_{j}\right|} \sum_{\mathbf{p} \in \mathcal{P}_{j}} \frac{\partial \mathbf{p}^{T}}{\partial \mathbf{T}_{i}} \frac{\partial}{\partial \mathbf{p}} \log L\left(\mathbf{p}\left(\mathbf{T}_{i}\right) \mid \theta_{i}\right)
$$

Computing the terms $\frac{\partial}{\partial \mathbf{p}} \log L\left(\mathbf{p} \mid \theta_{i}\right)$ requires us to define the GP likelihood $L\left(\mathbf{p} \mid \theta_{i}\right):=L_{x}\left(z \mid \theta_{i}\right)$. In this expression, $z$ refers the $z$-component of the transformed point $\mathbf{p}(\mathbf{T})=\left(x_{1}, x_{2}, z\right)$. Meanwhile, $L_{x}$ refers to the likelihood evaluated at the point $\mathbf{x}=\left(x_{1}, x_{2}\right)$. With the notation of [2], the likelihood of $z$ at $\mathbf{x}$ is given by

$$
\log L_{x}\left(z \mid \theta_{i}\right)=-\frac{1}{2} \log 2 \pi \sigma_{x}^{2}-\frac{1}{2 \sigma_{x}^{2}}\left(z-\boldsymbol{\alpha}^{T} \mathbf{k}_{x}\right)^{2} .
$$

The vector $\mathbf{k}_{x}$ consists of the kernel function evaluated at all the basis points of the GP. In our system, we use a squared exponential kernel but one may also use other kernels. We will therefore leave any derivations related to the kernel function as an exercise to the reader. If we assume Gaussian measurement noise in the $z$-axis with variance $\sigma_{0}^{2}$, the estimate uncertainty $\sigma_{x}^{2}$ at $\mathbf{x}$ is

$$
\sigma_{x}^{2}=\sigma_{0}^{2}+\mathbf{k}_{x}^{T} \mathbf{C k}_{x}+\mathbf{k}_{x}^{*} \text {. }
$$

The term $k_{x}^{*}$ is simply the kernel function at $x$ evaluated with itself. $\boldsymbol{\alpha}, \mathbf{C}$ are variables of the sparse GP method.

In this paper, we assume that the error in $z$ is negligible, leaving us to optimize the $x y$-position. We therefore proceed to derive the gradient of the likelihood with respect to $\mathbf{x}$. Since both $\mathbf{k}_{x}$ and $k_{x}^{*}$ depend on $\mathbf{x}$, we start by differentiating the uncertainty:

$$
\frac{\partial \sigma_{x}^{2}}{\partial \mathbf{x}}=\frac{\partial k_{x}^{*}}{\partial \mathbf{x}}+\frac{\partial \mathbf{k}_{x}^{T}}{\partial \mathbf{x}}\left(\mathbf{C}+\mathbf{C}^{T}\right) \mathbf{k}_{x} .
$$

With this result in hand, we look at Equation 3 to derive the full likelihood gradient

$$
\begin{aligned}
\frac{\partial}{\partial \mathbf{x}} \log L_{x}(z \mid \theta) & =-\frac{1}{2 \sigma_{x}^{2}} \frac{\partial \sigma_{x}^{2}}{\partial \mathbf{x}} \\
& +\frac{\partial \sigma_{x}^{2}}{\partial \mathbf{x}} \frac{1}{2 \sigma_{x}^{4}}\left(z-\boldsymbol{\alpha}^{T} \mathbf{k}_{x}\right)^{2} \\
& +\frac{1}{\sigma_{x}^{2}}\left(z-\boldsymbol{\alpha}^{T} \mathbf{k}_{x}\right) \frac{\partial \mathbf{k}_{x}^{T}}{\partial \mathbf{x}} \boldsymbol{\alpha}
\end{aligned}
$$

Looking at Equation 2, what remains is to compute the transform Jacobian $\frac{\partial \mathbf{p}^{T}}{\partial \mathbf{T}}$. Since $\mathbf{p}(\mathbf{T})$ is a simple $x y$ translation $\mathbf{p}(\mathbf{T})=\left(p_{x}+T_{x}, p_{y}+T_{y}, p_{z}\right)$, the Jacobian is $\frac{\partial \mathbf{p}^{T}}{\partial \mathbf{T}}=\left[\begin{array}{ll}\mathbf{I}_{2} & \mathbf{0}_{2 \times 1}\end{array}\right]$. With the likelihood gradient $\frac{\partial}{\partial \mathbf{T}_{i}} N L L_{i}\left(\mathcal{P}_{j}, \mathbf{T}_{i}\right)$ computed, we are ready to turn the problem of optimizing $c$ over to an non-linear optimizer.

\section{EXPERIMENTS}

We conduct experiments on the pockmarks [3] and pipeline datasets to validate compression of the maps, as well as loop closure alignment and filtering. To compute the alignment error between several submaps of points, we use the method of [13]. The uncertainty varies somewhat between the datasets. In the pipeline dataset, we have better initial position estimates as well as lower measurement noise, partly because the vehicle was flying closer to the sea floor. We therefore use slightly different measurement and motion model noise 


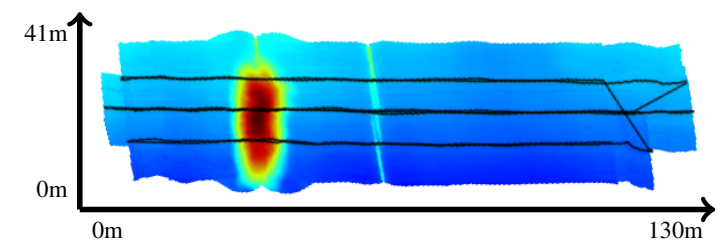

(a) Dead reckoning elevation, with hill and pipe.

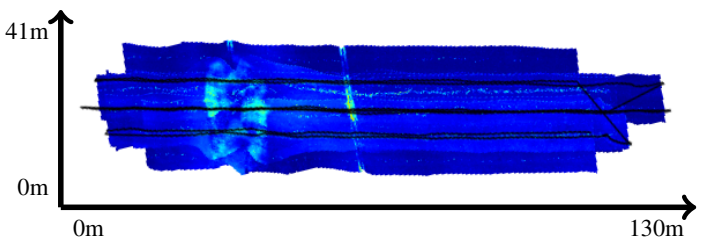

(c) GPGS Slam removes most of the alignment error.

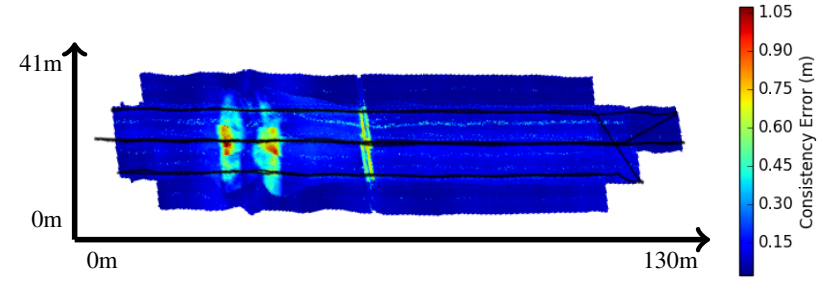

(b) Dead reckoning exhibits large errors on hill and pipe.

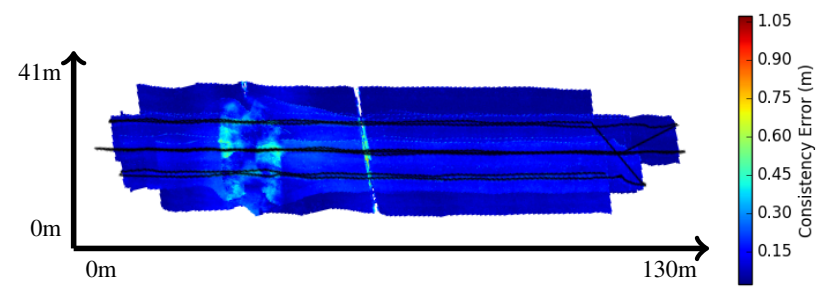

(d) GP filtering removes the noisy line along the middle.

Fig. 3: The top left map represents the fused elevation of the 7 submaps in the pipeline dataset, with estimates from DR. The others are consistency maps with the error from [13]. The errors get progressively better with slam and filtering, though the latter erroneously removes parts of the pipe in some submaps.

parameters in the two experiments. However, we use the same prior on the sea floor shape, i.e. the same squared exponential parameters $\left(l_{s e}, \sigma_{s e}^{2}\right)$, as seen in Table I.

\begin{tabular}{r|ccccc} 
Dataset & $\sigma_{1}^{2}$ & $\sigma_{0}^{2}$ & $l_{\text {se }}$ & $\sigma_{\text {se }}^{2}$ & $\tau$ \\
\hline Pockmarks & 0.04 & 0.03 & 7 & 1 & 0.3 \\
Pipeline & 0.01 & 0.01 & 7 & 1 & 0.3
\end{tabular}

TABLE I: Parameters used in the two experiments. Note that we use assume an IID motion model, $Q_{1}=\sigma_{1}^{2} \mathbf{I}$.

We always train the parameters $\theta_{i}$ of each submap using all of the original points $\mathcal{P}_{i}$, with no preprocessing. In the global alignment optimization of $c$, subsampling of the evaluated submap points is performed to reduce the run time of the algorithm. In both experiments, we keep only every 300th point for this purpose, dramatically reducing the necessary evaluations of $N L L_{i}$ and its corresponding gradient.

\section{RESUlTS}

In Table II, we see the compression results on the two datasets. The sizes of the point clouds are computed by saving them to disk in binary form, and the GP size is computed by storing its parameters, including basis vectors, in the same binary format. The point clouds are then reconstructed from the GPs by retrieving the mean altitude at a fixed lattice of $x y$-positions. We observe compression ratios of two to three orders of magnitude, with some small reconstruction error as a trade-off. If a higher precision is required, the method of [2] allows for this, in return for higher storage requirements.

\begin{tabular}{r|cc} 
& Pockmarks & Pipeline \\
\hline Number submaps & 17 & 7 \\
Compression (MB) & $75 \rightarrow 2.3$ & $962 \rightarrow 0.3$ \\
Map RMS Error [13] (m) & 0.19 & 0.12
\end{tabular}

TABLE II: Compression results. The RMS error was computed by sampling the Gaussian processes at fixed intervals, and comparing to the original submap clouds $\mathcal{P}_{i}$ using [13].

For both datasets, training the submap GPs takes less than a second, while global alignment of all submaps takes less than a minute with the mentioned subsampling. The pockmarks data was collected over approximately 140 minutes, and the pipeline data over 45 minutes. Our algorithm should therefore be able to run several times over the course of a mission, potentially at every loop closure.

In Figure 2 and Table III, we see the alignment error before and after applying SLAM and filtering on the pockmarks dataset. The error is reduced by our submap alignment, and reduced further by removing some of the points based on the likelihood threshold. By comparing the track lines of the original DR estimate in Figure 2a with those of the refined estimate in Figure 2b, we see that horizontal lines are shifted further to the right as we go up the $y$-axis. This indicates that the DR drifted along the figure $\mathrm{x}$-axis during the course of the mission, 
maybe due to currents. Therefore, at the end of the run, the DR estimate has seemingly drifted tens of meters. This example highlights one of the strong points of the proposed algorithm. While there will initially be large transform errors between matched submaps $(i, j) \in \mathcal{M}$ that were collected far apart in time, registration of the other matches will gradually force them together. Once the transform error has been reduced, even those pairs start contributing to a refined alignment estimate, since the registration is performed online with optimization.

\begin{tabular}{r|cc} 
Map RMS Error [13] $(\mathrm{m})$ & Pockmarks & Pipeline \\
\hline Initial (DR) & 0.73 & 0.17 \\
GPGS Slam & 0.49 & 0.15 \\
GPGS Slam + Filtering & 0.47 & 0.14
\end{tabular}

TABLE III: SLAM and filtering results. Errors are computed by transforming the original points by the optimized transforms.

In Figure 3, we see results from the pipeline dataset. Submap alignment removes much of the error, which seems to stem mostly from drift along the vehicle direction. However, the relative improvement presented in Table III is smaller for this dataset, likely due to the large flat areas. Before filtering there is also additional noise remaining that can be attributed to the sensor. In particular, one can clearly see consistent noise along some beam angles in Figures 3b-c. The filtering, showcased in Figure 3d, manages to remove these kinds of errors by utilizing the smoothness prior within the submaps. It also filtered some actual abrupt changes in the sea floor, in this case parts of a pipeline. Note that all parts still persisted in some of the submaps, giving a complete bathymetry map of the area. Nonetheless, it highlights one of the potential pitfalls of the method.

\section{CONClusions \& Future WORK}

In conclusion, sparse GPs is a flexible tool that has multiple advantages as a representation of bathymetry data. Here we have presented several novel use cases, with promising results on real world data. Notably, the method can reduce storage requirements by orders of magnitude, while maintaining maps that can be used for Terrain-Aided SLAM. Indeed, the main advantage over previous work is the capability to scale the storage and speed to allow for large globally consistent bathymetric maps, while maintaining high precision.

A pitfall of general GPs as a representation becomes apparent when the smooth sea floor prior is not valid. This may happen for example with steep rocks, or with a pipeline, as seen in the experiments. At present, we accept the inability to represent abrupt changes, as the best fit of the alignment is likely to be approximately correct. However, with finer detail in those areas, alignment could be improved. In future work, one could apply a scheme similar to our likelihood filtering to identify larger homogeneous areas of change. One could then represent those areas using other methods, or with adaptive non-stationary kernels, as suggested in [15].

\section{ACKNOWLEDGMENTS}

The authors want to thank Stefan Williams, Oscar Pizarro and colleagues for allowing us to use the pockmarks dataset for our evaluation. This work was supported by Stiftelsen för Strategisk Forskning (SSF) through the Swedish Maritime Robotics Centre (SMaRC) (IRC15-0046).

\section{REFERENCES}

[1] V. Bichucher, J. M. Walls, P. Ozog, K. A. Skinner, and R. M. Eustice, "Bathymetric factor graph slam with sparse point cloud alignment," in OCEANS'15 MTS/IEEE Washington, pp. 1-7, IEEE, 2015.

[2] L. Csató and M. Opper, "Sparse on-line gaussian processes," Neural computation, vol. 14, no. 3, pp. 641-668, 2002.

[3] S. Barkby, S. B. Williams, O. Pizarro, and M. V. Jakuba, "Bathymetric particle filter slam using trajectory maps," The International Journal of Robotics Research, vol. 31, no. 12, pp. 1409-1430, 2012.

[4] I. Nygren and M. Jansson, "Terrain navigation for underwater vehicles using the correlator method," IEEE Journal of Oceanic Engineering, vol. 29, pp. 906-915, July 2004.

[5] B. Claus and R. Bachmayer, "Terrain-aided navigation for an underwater glider," Journal of Field Robotics, vol. 32, no. 7, pp. 935-951, 2015.

[6] L. Zhou, X. Cheng, and Y. Zhu, "Terrain aided navigation for autonomous underwater vehicles with coarse maps," Measurement Science and Technology, vol. 27, no. 9, p. 095002, 2016.

[7] C. Roman and H. Singh, "Improved vehicle based multibeam bathymetry using sub-maps and slam," in 2005 IEEE/RSJ International Conference on Intelligent Robots and Systems, pp. 3662-3669, Aug 2005.

[8] P. V. Teixeira, M. Kaess, F. S. Hover, and J. J. Leonard, "Underwater inspection using sonar-based volumetric submaps," in Intelligent Robots and Systems (IROS), 2016 IEEE/RSJ International Conference on, pp. 4288-4295, IEEE, 2016.

[9] S. Barkby, S. B. Williams, O. Pizarro, and M. V. Jakuba, "A featureless approach to efficient bathymetric slam using distributed particle mapping," Journal of Field Robotics, vol. 28, no. 1, pp. 19-39, 2011.

[10] S. Barkby, S. B. Williams, O. Pizarro, and M. V. Jakuba, "Bathymetric slam with no map overlap using gaussian processes," in 2011 IEEE/RSJ International Conference on Intelligent Robots and Systems, pp. 1242-1248, Sept 2011.

[11] P. Norgren and R. Skjetne, "A multibeam-based slam algorithm for iceberg mapping using auvs," IEEE Access, 2018.

[12] M. Bleier, A. Dias, A. Ferreira, J. Pidgeon, J. Almeida, E. Silva, K. Schilling, and A. Nüchter, "Signed distance function based surface reconstruction of a submerged inland mine using continuous-time slam," IFAC-PapersOnLine, vol. 50, no. 1, pp. 1139-1144, 2017.

[13] C. Roman and H. Singh, "Consistency based error evaluation for deep sea bathymetric mapping with robotic vehicles," in Robotics and automation, 2006. ICRA 2006. Proceedings 2006 IEEE international conference on, pp. 3568-3574, Ieee, 2006.

[14] S. Agarwal, K. Mierle, and Others, "Ceres solver." http: / / ceres-solver.org.

[15] T. Lang, C. Plagemann, and W. Burgard, "Adaptive nonstationary kernel regression for terrain modeling.," in Robotics: Science and Systems, vol. 6, 2007. 